\title{
Intelligent signal detectors with random moment of appearance in rail lines monitoring systems
}

\author{
Nazirjon Aripov ${ }^{*}$, Azamat Sadikov, and Saidazim Ubaydullayev \\ Tashkent State Transport University, Tashkent, Uzbekistan,
}

\begin{abstract}
The article discusses the issues of building intelligent receivers of pulsed signals with an unknown arrival time without restrictions on the value of the signal-to-noise ratio under the influence of a complex of interfering factors. Using recurrent methods for detecting disturbances of random processes and an algorithm of cumulative sums, the problem of synthesizing algorithms for detecting Markov signals with random moments of appearance against the background of various combinations of destabilizing factors acting in control systems for the states of rail lines in normal and shunt operating modes is solved. To assess the efficiency of detecting a random signal by the method of cumulative sums, statistical modeling of the specified algorithm was carried out.
\end{abstract}

\section{Introduction}

When building intelligent receivers of pulse signals with an unknown arrival time without restrictions on the value of the signal-to-noise ratio under the influence of a complex of interfering factors, good results are obtained using the mathematical apparatus for detecting the disorder of random processes [1-3]. The disorder is understood as an abrupt change in the properties of a random process. This can be, for example, the scalar parameter of the probability density distribution $w\left(y_{h}\right)$ of observation $y_{h}$, where $h$ is the number of the time sample of the signal. The issue of signal detection is solved based on the analysis of the realizations of the input action that are successively received at the input of the receiver. Since this increases the amount of memory required to memorize all observations $h$ received at an arbitrary moment, the question arises of finding recurrent algorithms. Such algorithms are based on sufficient statistics that allow recalculating the previous values of observations taking into account the newly received ones $[4,5]$.

\section{Methods}

\footnotetext{
${ }^{*}$ Corresponding author: aripov1110@gmail.com
} 
Of the recurrent methods for detecting single disturbances, the cumulative sum algorithm (CSA) with a reflecting screen has found wide application [6]. It is a modified Wald sequential analysis [7]. Discrepancy detection is based on a comparison at the $(h+1)$ - the step of some decision statistics $S_{h+1}$, with fixed thresholds $\mathrm{U}_{\Pi в}$ and $\mathrm{U}_{\Pi н}$ :

$$
S_{h+1}=\left[S_{h}+\Omega_{h+1}\right]_{U_{\Pi H}^{+}} \geq U_{\Pi B}
$$

where the sign "+" means the setting of zero of the cumulative sum at the moments of time $h(t) ; t=1,2, \ldots, h(t)$ are successive times when $S_{h+i}=0$;

$$
\Omega_{h+1}=\ln \frac{w\left(y_{h+1} \mid \theta_{2}\right)}{w\left(y_{h+1} \mid \theta_{1}\right)}
$$

The $\mathrm{U}_{\Pi \mathrm{B}}$ threshold is set according to the required probability of a false detection $\mathrm{F}$ and determines the probability of skipping a breakdown. Thus, if at the $h$ - the step $S_{h}>U_{P V}$ , the hypothesis $\mathrm{H}_{1}$ is accepted (there is a disorder): $\theta=\theta_{2}$. If $S_{h}<-U_{P N}$, the hypothesis $\mathrm{H}_{0}$ is accepted (no discord): $\theta=\theta_{1}$. Otherwise, if $-U_{P N}<S_{h}<U_{P N}$, $(h+1)-$ the observation. However, since this violates the assumption that the entire sample belongs to the hypothesis $\mathrm{H}_{1}$ or $\mathrm{H}_{0}$, then in the case when the hypothesis $\mathrm{H}_{0}$ is accepted at the $h$ - the step, the cumulative sum is zeroed at the next step, etc., $S_{h+1}=0$ $[8-10]$.

\section{Results and Discussions}

Let us consider the problem of synthesizing algorithms for detecting Markov signals with random moments of appearance against the background of various combinations of destabilizing factors acting in control systems for the states of rail lines [11].

a. Case 1:

Let there be a sequence $\left\{y_{h}\right\}, h=\overline{1, H}$

$$
y_{h}=\theta_{\tau} s\left(\lambda_{c h}, \xi_{h}\right)+\eta\left(\xi_{h}\right)+n_{h}
$$

where $\lambda_{c h}, \xi_{h}$ are the parameters of the useful signal $s\left(\lambda_{c h}, \xi_{h}\right)$ and interference $\eta\left(\xi_{h}\right)$ , which are homogeneous discrete Markov chains with $l$ - and $r$ - states, transition $\mathrm{p}_{\alpha \beta}, \mathrm{p}_{\gamma \chi}$ and initial $p\left(\lambda_{1}\right)$ - and $p\left(\xi_{1}\right)$ - probabilities. The sequences $\left\{\lambda_{c h}\right\}$ and $\left\{\xi_{h}\right\}$ are assumed to be statistically independent. Impulse noise $\eta\left(\xi_{h}\right)$ is a random process emissions caused by instability of the train shunt resistance [12]. Fluctuation noise $\left\{n_{h}\right\}$ is a correlated random process with a known conditional probability density $w_{P}\left(n_{h} \mid n_{h}-1\right)$. 
Two hypotheses are considered regarding the parameter $\theta_{\tau}$ :

$$
\left.\begin{array}{l}
H_{0}: \theta_{\tau}=\theta_{1}=0 ; 0 \leq h \leq H ; \\
H_{1}: \theta_{\tau}=0 ; 0 \leq h \leq \tau ; \\
\theta_{\tau}=\theta_{2}=1 ; \tau \leq h \leq \tau+T ; \\
\theta_{\tau}=0 ; \tau+T<h \leq H
\end{array}\right\}
$$

where $T$ is the signal duration.

The decision about the disorder is made at the moment of time $h^{*}$ satisfying the condition [13]

$$
h^{*}=\inf \left\{h \geq 1: S_{h} \geq U_{P V}\right\}
$$

If we restrict ourselves to considering the case of a high SIR and assume that the duration of the pulse signal $T$ is such that $m h^{*}<\tau+T$, then the detection of a pulse of limited duration will be equivalent to recording a disorder of a random process (detection of the leading edge of the pulse) $[14,15]$. As a result, the detection problem is solved by forming a cumulative sum:

$$
S_{h+1}=\left\{S_{h}+\ln I_{h+1}\left[y_{h+1}, w_{P}\left(n_{h}\right), w_{h}\left(\lambda_{c}^{\beta}, \xi^{\gamma}\right)\right]\right\}_{h(t)}^{+}
$$

where

$$
\left.\begin{array}{c}
I_{h+1}(.)=\frac{\sum_{\alpha, \beta}^{l} \sum_{\gamma, \chi}^{r} w_{P 1} p_{\alpha \beta} p_{\gamma \chi} w_{1 h}\left(\lambda_{c}^{\beta}, \xi^{\gamma}\right)}{\sum_{\gamma \chi}^{r} w_{P 0} p_{\gamma \chi} w_{0 h}\left(\xi^{\gamma}\right)} \\
w_{P 1}=w_{P}\left[y_{h+1}-s\left(\lambda_{c h+1}^{\alpha}, \xi_{h+1}^{\gamma}\right)-\eta\left(\xi_{h+1}^{\chi}\right) \mid y_{h}-s\left(\lambda_{c h}^{\alpha}, \xi_{h}^{\gamma}\right)-\eta\left(\xi_{h}^{\chi}\right)\right] \\
w_{P 0}=w_{P}\left[y_{h+1}-\eta\left(\xi_{h+1}^{\chi}\right) \mid y_{h}-\eta\left(\xi_{h}^{\chi}\right)\right]
\end{array}\right\}
$$

The posterior joint probability distribution densities (PDD) of the values of the signal and noise parameter $w_{1 h}\left(\lambda_{c}{ }^{\beta}, \xi^{\gamma}\right)=p\left(\lambda_{c}{ }^{\beta}, \xi^{\gamma} \mid y_{h(t)}^{h}, \theta_{2}\right)$ and one noise $w_{0 h}\left(\xi^{\gamma}\right)=p\left(\xi^{\gamma} \mid y_{0}^{h}, \theta_{1}\right)$ satisfy the following recurrent equations: 


$$
\begin{aligned}
& w_{1 h+1}\left(\lambda_{c}^{\alpha}, \xi^{\chi}\right)=\frac{w_{P 1} \sum_{\beta, \gamma}^{l, r} p_{\alpha, \beta} p_{\gamma, \chi} w_{1 h}\left(\lambda_{c}^{\beta}, \xi^{\gamma}\right)}{\sum_{\alpha, \beta}^{l} \sum_{\gamma, \chi}^{r} w_{P 1} p_{\alpha, \beta} p_{\gamma, \chi} w_{1 h}\left(\lambda_{c}^{\beta}, \xi^{\gamma}\right)} \\
& w_{0 h+1}\left(\xi^{\chi}\right)=\frac{w_{P 0} \sum_{\gamma=1}^{r} p_{\gamma, \chi} w_{0 h}\left(\xi^{\gamma}\right)}{\sum_{\gamma}^{l} \sum_{\chi}^{r} w_{P 0} p_{\gamma, \chi} w_{0 h}\left(\xi^{\gamma}\right)} \\
& \alpha, \beta=\overline{1, l ; \gamma, \chi}=\overline{1, r}
\end{aligned}
$$

The recurrent algorithm for the formation of a posteriori PDD allows not to keep in memory all the previously received count of signals $\mathrm{y}_{1}, \mathrm{y}_{2}, \ldots, \mathrm{y}_{\mathrm{h}}$, since they are included in the PDD $w_{1 h}\left(\lambda^{\alpha}, \xi^{\gamma}\right)$ already formed at the previous step. This algorithm can be divided into two procedures performed in stages. Let us analyze this by the example of the PDD $w_{0 h+1}\left(\xi^{\chi}\right)$. At the first stage of calculating the PDD, extrapolation of the previous posteriori PDD is carried out by the step size $T_{0}$ to the moment of the next observation [16, 17]. The extrapolated estimate of the probability distribution density has the form:

$$
w_{0 h+1 \ni}\left(\xi^{\chi}\right)=\sum_{\gamma=1}^{r} p_{\gamma \chi} w_{0 h}\left(\xi^{\gamma}\right)
$$

At the second stage, a new posteriori PDD is formed based on the extrapolated estimate $w_{0 h+1 \jmath}\left(\xi^{\chi}\right)$ and the next observation $y_{h+l}$. As a result, we get:

$$
w_{0 h+1}\left(\xi^{\chi}\right)=\frac{w_{P 0} w_{0 h+1 \jmath}\left(\xi^{\chi}\right)}{\sum_{\gamma=1}^{r} w_{P 0} w_{0 h+1 \ni}\left(\xi^{\chi}\right)}
$$

The last entry allows us to consider the extrapolated PDD $w_{0 h+1 \jmath}\left(\xi^{\chi}\right)$ as a priori with respect to the next observation.

Similar ratios can be written for posteriori PDD $w_{1 h+1}\left(\lambda_{c}^{\alpha}, \xi^{\chi}\right)$.

At the moment of zeroing the cumulative sums $\mathrm{h}(\mathrm{t})$, each time, it is necessary to form a new initial PDD $w_{1 h(t)}\left(\lambda_{h(t)}^{\beta}, \xi_{h(t)}^{\gamma}\right)$.

From the above algorithm, we single out a particular case of signal detection against the background of interference with independent values [18]. For this, in the presented formulas, instead of conditional PDD and calculated based on expressions (4), it is sufficient to substitute one-dimensional PDD: 


$$
\begin{gathered}
w_{n 1}=w_{n}\left[y_{h+1}-s\left(\lambda_{c h+1}^{\alpha}, \xi_{h+1}^{\gamma}\right)-\eta\left(\xi_{h+1}^{\chi}\right)\right], \\
w_{n 0}=w_{n}\left[y_{h+1}-\eta\left(\xi_{h+1}^{\chi}\right)\right]
\end{gathered}
$$

\section{b. Case 2}

Let the observation have access to the sequence

$$
y_{h}=\theta_{\tau}+\xi_{h}+n_{h}
$$

where $\theta_{\tau}$ is the signal amplitude varying at an unknown time moment $\tau ; n_{h}$ is fluctuation noise with a known non-Gaussian PDD $w_{n}\left(n_{h}\right)$, the parameters of which change at the time of the breakdown; impulse noise described by a Markov chain with discrete time and a finite number of inconsistent states $\xi^{(1)}, \xi^{(2)}, \ldots \xi^{(l)}$. The random variables $\{\xi h\}$ are given by the matrix of transition probabilities:

$$
\left\|p\left\{\xi_{h+1}=\xi^{\alpha} \mid \xi_{h}=\xi^{\beta}\right\}\right\|=\left\|p_{\alpha \beta}\right\|, \alpha, \beta=\overline{1, l}
$$

vector of initial probabilities $\left\|p_{1}, p_{2}, \ldots, p_{l}\right\|$ and vector of initial values $\left\|\xi_{0}^{(1)}, \xi_{0}^{(2)}, \ldots, \xi_{0}^{(l)},\right\|$

Obviously, (6) is a special case of (2) for $s()=.l ; \eta(\xi)=\xi$. Two hypotheses are considered regarding the parameter $\theta_{\tau}$ :

$$
\begin{aligned}
& H_{0}: \theta_{\tau}=\theta_{1}, w_{P}\left(n_{h}, \sigma_{p}^{2} \neq 0\right), h=\overline{1, H} \\
& H_{1}: \theta_{\tau}=\theta_{1}, w_{P}\left(n_{h}, \sigma_{p}^{2} \neq 0\right), h=\overline{1, \tau-1} \\
& \theta_{\tau}=\theta_{2}, w_{P}\left(n_{h}, \sigma_{p}^{2}=0\right), h=\overline{\tau, H}
\end{aligned}
$$

For the case under consideration, we write the CSA in the form [19, 20]:

$$
S_{h+1}=\left\{S_{h}+\ln I_{h+1}\left[y_{0}^{h+1}, w\left(\xi_{h} \mid y_{0}^{h}\right), \theta_{\tau}\right]\right\}^{+}
$$

where $y_{0}{ }^{h+1}=\left\{y_{1}, y_{1}, \ldots, y_{h+1},\right\}$ is vector of observed realization; $w\left(\xi_{h} \mid y_{0}{ }^{h}\right)$ is posteriori PDD of the unobservable Markov component $\xi_{h}$. Function:

$$
I_{h+1}[.]=\frac{\sum_{\xi_{h}, \xi_{h+1}}^{l} w_{P 2}\left(y_{h+1} \mid \xi_{h+1}, \theta_{\tau}=\theta_{2}\right) w\left(\xi_{h} \mid y_{0}^{h}, \theta_{\tau}=\theta_{2}\right) p_{\alpha \beta}}{\sum_{\xi_{h}, \xi_{h+1}}^{l} w_{P 1}\left(y_{h+1} \mid \xi_{h+1}, \theta_{\tau}=\theta_{1}\right) w\left(\xi_{h} \mid y_{0}^{h}, \theta_{\tau}=\theta_{1}\right) p_{\alpha \beta}}
$$

characterizes the change in the likelihood ratio at the $(h+1)-$ the step, where $w_{n 1}()=.w_{n}\left(n_{h}, \sigma_{p}^{2}=0\right), w_{n 2}()=.w_{n}\left(n_{h}, \sigma_{p}^{2} \neq 0\right)$. 
The initial posteriori PDD are calculated as follows:

$$
w\left(\xi_{1} \mid y_{1}, \theta_{i}\right)=\frac{w_{P 2}\left(y_{1}-\xi_{2}-\theta_{i}\right) p_{\alpha}}{\sum_{\alpha=1}^{l} w_{P 1}\left(y_{1}-\xi_{1}-\theta_{i}\right) p_{\alpha}}, \alpha=\overline{1,2}
$$

In what follows, calculations using the CSA are performed similarly to case 1 .

c. Evaluation of the efficiency of algorithms for detecting signals with an unknown appearance time by the CSA method against the background of a complex of destabilizing factors

Let us consider specific examples of the synthesis of algorithms for detecting signals by the CSA method and evaluating their work efficiency. Let it be required to detect a change in the voltage amplitude $\theta_{\tau}$ at the receiver's input under the action of white Gaussian noise $n_{h}, n_{h} \in N\left(0, \sigma^{2}\right)$. Observationally available implementation:

$$
y_{h}=\theta_{\tau}+n_{h} .
$$

Two hypotheses are considered regarding the amplitude of the useful signal $\theta_{\tau}$ :

$$
\begin{aligned}
& H_{0}: \theta_{\tau}=\theta_{1} ; n_{h} \in N\left(0, \sigma^{2}\right) ; \\
& H_{1}: \theta_{\tau}=\theta_{1} ; n_{h} \in N\left(0, \sigma^{2}\right) ; \\
& \theta_{\tau}=\theta_{2} ; n_{h} \in N\left(0, \sigma^{2}\right)
\end{aligned}
$$

Using the cumulative sum algorithm [2], we write:

$$
S_{h+1}=\left\{S_{h}+\ln \frac{w_{N}\left(y_{h+1} \mid \theta_{2}\right)}{w_{s h}\left(y_{h+1} \mid \theta_{1}\right)}\right\}^{+} \geq U_{P V}, S_{0}=0,
$$

where $w_{H}, w_{u}$ are the probability distribution densities of the signal $y_{h}$ in normal and shunt modes:

$$
\begin{aligned}
& w_{s h}\left(y_{h+1} \mid \theta_{1}\right)=\frac{1}{\sqrt{2 \pi \sigma^{2}}} \exp \left\{-\frac{\left(y_{h+1}-\theta_{1}\right)^{2}}{2 \sigma^{2}}\right\}, \\
& w_{N}\left(y_{h+1} \mid \theta_{2}\right)=\frac{1}{\sqrt{2 \pi \sigma^{2}}} \exp \left\{-\frac{\left(y_{h+1}-\theta_{2}\right)^{2}}{2 \sigma^{2}}\right\}
\end{aligned}
$$

To assess the efficiency of detecting a random signal by the method of cumulative sums for this observation, statistical modeling of the specified algorithm was carried out. Sampled values of the realization $y_{h}$ were fed to the input of the receiving device, in which, after a certain number of reports, the amplitude of the useful signal changed. The amplitude of the useful signal $\theta$ was considered known: $\theta_{1}=4.8 \mathrm{~V}$ in the shunt mode, $\theta_{2}=6.572 \mathrm{~V}-$ in the normal mode and unchanged in the observation interval. Figure 1 illustrates the wave diagrams of the input process $y_{h}$ and the behavior of the cumulative sum $S_{h}$; the detection of a disorder from the moment the pulse front appears and the solution of the receiver $\mathrm{H}$. Parameters of white Gaussian noise, respectively $\sigma=0.32 \mathrm{~V}$.

In total, 31500 realizations of the described random process were considered: 13500 realizations in the normal one; 18000 realizations in shunt mode. Based on the results of 
statistical modeling, estimates of the probabilities of missing a useful signal $P_{P R}$ in the normal mode and false signal detection in the shunt mode $P_{L O}$

$$
\begin{gathered}
P_{P R}=\frac{n_{P R}}{N_{N}}, \\
P_{L O}=\frac{n_{L O}}{N_{S h}}
\end{gathered}
$$

where $n_{P R}, n_{L O}$ is the number of cases of missing and false signal detection; $N_{N}, N_{S h}$ is total number of realizations in normal and shunt modes.

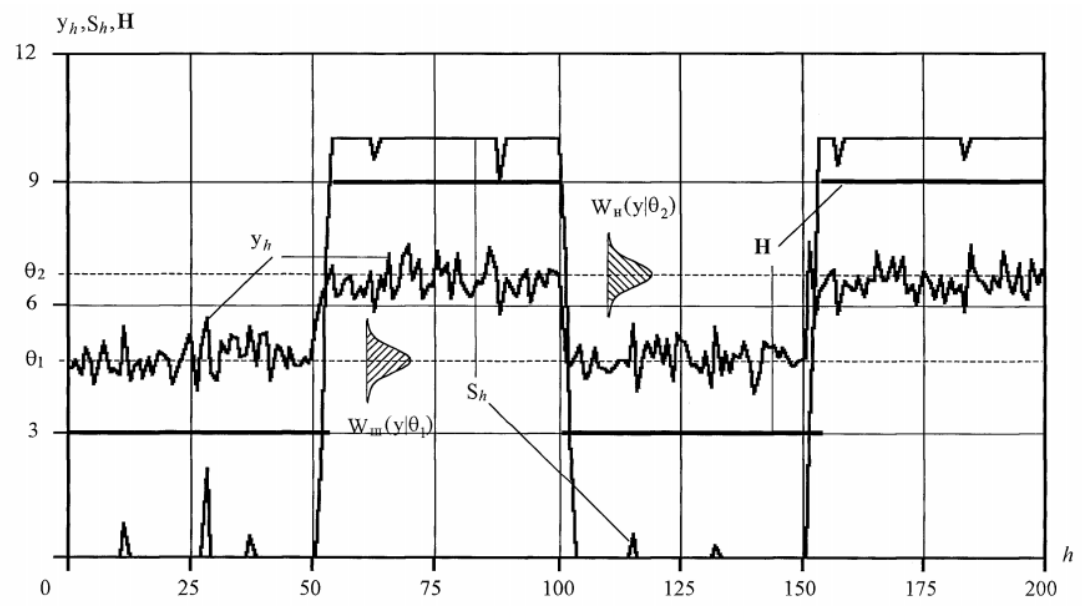

Fig.1. Wave diagrams of the observed realization $y_{h}$, the cumulative sum Sh and the solution of the receiver $\mathrm{H}$

Figure 2, $a$ illustrates the dependence of the probability of missing a useful signal $P_{P R}$ on the root-mean-square deviation of the noise $\sigma$ for various thresholds of disorder $U_{P R}$. From the analysis of the obtained dependences, it follows that with an increase in the standard deviation of the interference $\sigma$, the probability of missing the useful signal $P_{P R}$ increases.

In figure $2, b$ shows the probabilities of false detection of $R_{L O}$ from the standard deviation of white Gaussian noise $\sigma$ for different values of the thresholds of the mismatch $U_{P V}$. With an increase in the value of the threshold $U_{P V}$, the probability of false detection $R_{L O}$ decreases.
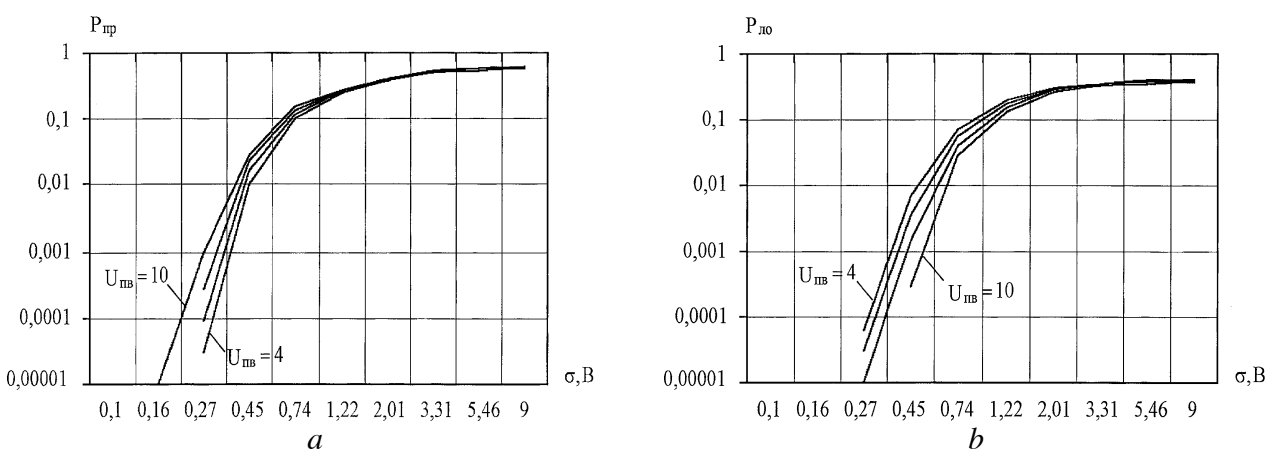
Fig.2. Dependences of the probabilities of missing $P_{P R}$ (a) and false detection $P_{L O}$ (b) on the values of the standard deviation of fluctuation noise $\sigma$

Let us consider the case when, in the shunt mode, both fluctuation noise $n_{h}$ and impulse noise $\xi_{h}$, caused by the instability of the resistance of the train shunt. Let $\xi_{h}$ be the impulse component described by a Markov chain with discrete time and a finite number of inconsistent states. The random variables $\left\{\xi_{h}\right\}$ are given by the matrix of transition probabilities

$$
\left\|P_{\alpha \beta}\right\|=\left\|P\left\{\xi_{h+1}=\xi^{\alpha} \mid \xi_{h}=\xi^{\beta}\right\}\right\|, \alpha, \beta=\overline{1,2}
$$

vector of initial probabilities $\left\|P_{1} P_{2}\right\|$ and vector of initial values $\left\|\xi_{0}^{(1)} \xi_{0}^{(2)}\right\|$. The implementation at the receiver input has the form:

$$
y_{h}=\theta_{\tau}+\xi_{h}+n_{h}
$$

Using the cumulative sum algorithm, we write:

$$
S_{h+1}=\left\{S_{h}+\ln I_{h+1}\left[y_{0}^{h+1}, w\left(\xi_{h} \mid y_{0}^{h}\right), \theta_{\tau}\right]\right\}^{+} \geq U_{P V}, S_{0}=0
$$

where $\theta_{1}=4.8 B, \theta_{2}=6.572 B$;

$y_{0}^{h+1}=\left\{y_{1}, y_{2}, \ldots, y_{h+1}\right\}$ is vector of observed realization;

$w\left(\xi_{h} \mid y_{0}{ }^{h}\right)$ is the posterior probability density of the unobservable component $\xi_{h}$;

$$
I_{h+1}[\cdot]=\frac{w_{s h}\left(y_{h+1} \mid \theta_{\tau}=\theta_{2}\right)}{\sum_{\xi_{h}, \xi_{h+1}}^{l} w_{s h}\left(y_{h+1} \mid \xi_{h+1}, \theta_{\tau}=\theta_{1}\right) w\left(\xi_{h} \mid y_{0}^{h}, \theta_{\tau}=\theta_{1}\right) P_{\alpha \beta}}
$$

\section{Conclusions}

In the simulation, 18000 realizations were considered in shunt mode. As a result of the research, the dependence of the probabilities of false detection $P_{\text {ЛО }}$ on the variance of interference. With an increase in the value of the root-mean-square deviation of the noise $\sigma$, the probability of false detection $P_{л о}$ increases. 
a) with two stable states

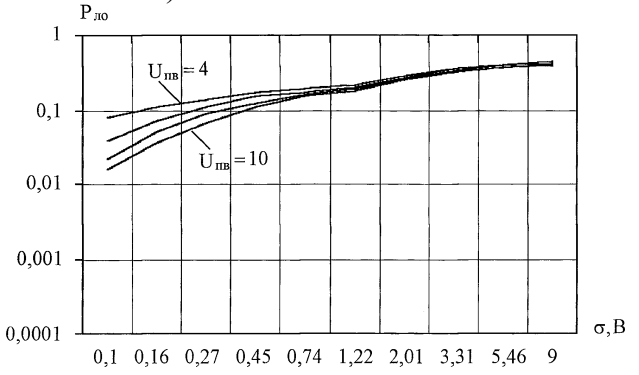

b) with three stable states

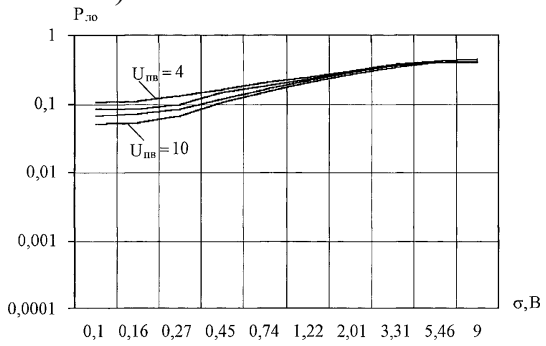

Fig.3. Dependences of the probabilities of false detection under the influence of a complex of fluctuation noise and an impulse component of interference caused by the instability of the resistance of the train shunt of a two-axle railcar

a) with two stable states

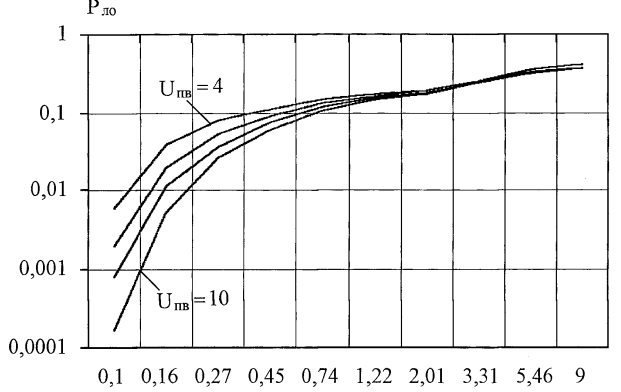

b) with three stable states

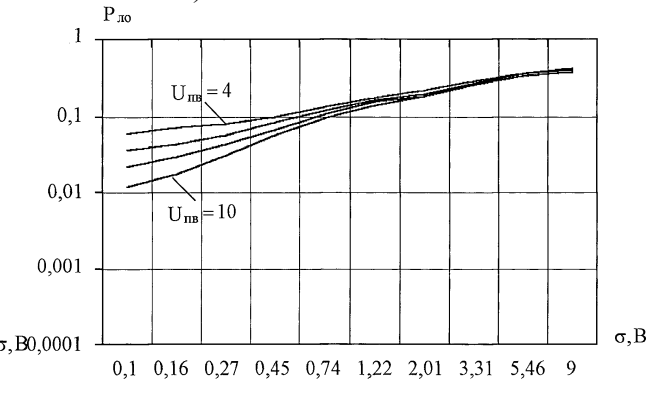

Fig. 4. Dependences of the probabilities of false detection under the influence of a complex of fluctuation noise and an impulse component of interference caused by the instability of the resistance of the train shunt of a three-axle railcar

a) with two stable states

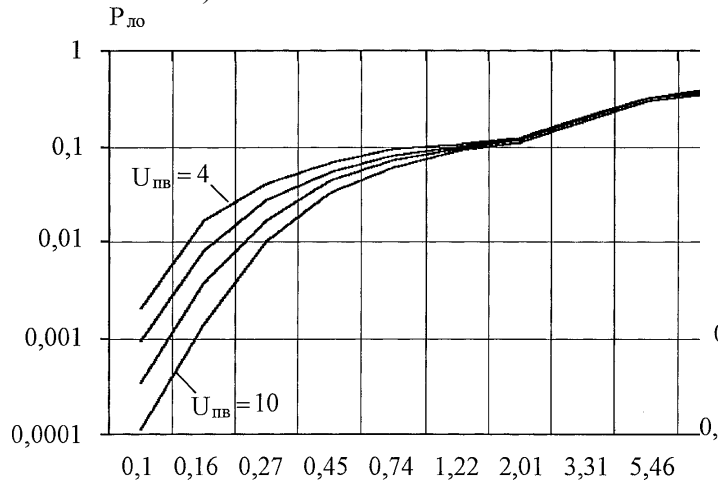

b) with three stable states
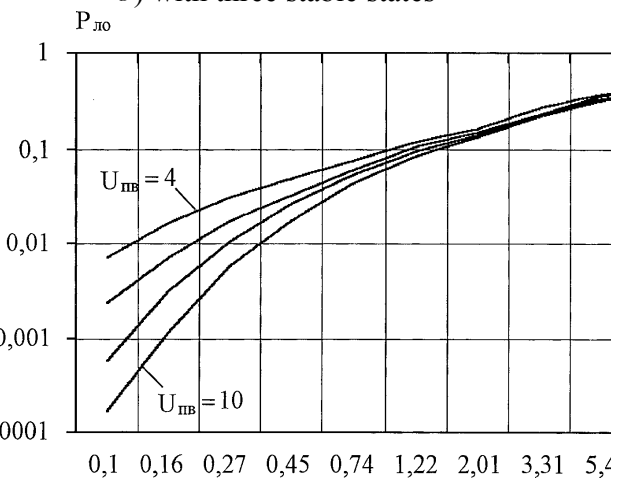

Fig. 5. Dependences of the probabilities of false detection under the influence of the complex of fluctuation noise and the impulse component of the noise caused by the instability of the resistance of the train shunt of the diesel locomotive

$d$. In figure 3 shows the probabilities of false detection $\mathrm{P}_{\text {ло }}$ from the standard deviation of white Gaussian noise $\sigma$ when in the shunt mode, impulse noise is affected due to the instability of the resistance of the train shunt of a biaxial railcar for various states of the tread surface: $\mathrm{a}$ - clean and $\mathrm{b}$ - contaminated. 
e. Figure 4 illustrates the dependence of the probabilities of false detection $\mathrm{P}_{\text {ло }}$ when in the shunt mode there is interference caused by the instability of the resistance of the train shunt of the TU-5 diesel locomotive on the section with a clean rolling surface - $a$ and with a rust-covered layer - $b$.

$f$. In figure 5 shows the probabilities of false detection $\mathrm{P}_{\text {ло }}$ from the standard deviation of white Gaussian noise $\sigma$ when in the shunt mode there is interference caused by the instability of the resistance of the train shunt of a triaxial railcar for various states of the rolling surface: $\mathrm{a}$ - clean and $\mathrm{b}$ - contaminated.

\section{References}

1. Belyakov I.V. Theory and methods of implementing adaptive systems for monitoring the states of rail lines, DissDoct. tech. Sciences: 05.22.08 / MGUPS (MIIT) .- M., p 441.(1996).

2. Zhiglyavsky A.A., Kraskovsky A.E. Detection of disorder of random processes in problems of radio engineering. L .: Publishing house of the Leningrad University, $p$ 224. (1988)

3. Nikiforov I.V. Sequential detection of changes in properties, time series. -M .: Nauka, p 200. (1983).

4. Aripov N.M., Rikhsiev D.Kh. Microprocessor technologies in railway automation and telemechanics systems, International scientific and practical conference "EurasiaScience". Moscow, October 31, (2018).

5. Aripov N., Aliyev R., Baratov D.X., Ametova E.K. Features of construction of systems of railway automatics and telemechanics at the organization of high-speed traffic in the republic of Uzbekistan. 9th international scientific conference Transbaltica 2015 / Vilnius, 7-8 may, 2015. Procedia Engineering (134) p. 175 - 180. (2016)

6. Azizov A.R., Sadikov A.N. Development of a scheme for linking a microprocessor pulse relay with existing systems of railway automation and telemechanics. Republican scientific and technical conference with the participation of foreign scientists "Resource-saving technologies of automation, electrical communication and power supply of railway transport." - Tashkent, TashIIT. pp. 176-178. (2017).

7. Azizov A.R., Yuldashev Sh.M., Sadikov A.N. Modeling and optimization of the algorithm of the electromechanical impulse relay, Bulletin of transport of the Volga region, November-December. (4). ( 2018)

8. James Peterson. Petri Net Theory and the Modeling of Systems. University of Texas at Austin. Prentice Hall, Inc., Englewood Cliffs,N.J. p 263. (19840.

9. Soroko V.I. Relay railway automation and remote control. -M.: NPF PLANETA, - p 696. (in Russian) (2002).

10. Microprocessor interlocking systems: Ed. VL Sapozhnikova: -M: State Educational Institution "Educational and Methodological Center for Education in Railway Transport".p 398. (in Russian)(2008).

11. Nevarov P.A. Model for assessing the reliability of railway automation systems and remote control, Modern problems of improving the work of railway transport: intercollegiate collection of scientific papers. - M.: MIIT, 2009. pp. 22-26. (2009)

12. Gorelik A.V. Methods for analyzing the operational reliability and safety of railway automation and remote control systems A.V. Gorelik, P.A. Nevarov, N.A. Taradin // Reliability and quality: proceedings of the International Symposium. - Penza: Information and Publishing Center of PenzSU, 2009. - pp. 230-234. (2009). 
13. Gorelik A.V. Methods for analyzing the reliability and efficiency of functioning of the systems of railway automation and remote control, A.V. Gorelik, P.A. Nevarov, I.A. Zhuravlev, N.A. Taradin // Science and technology of transport (3). pp. 88-93. (2011)

14. Goce L. Arsov The 40th Anniversary of the Simulation Program with Integrated Circuit Emphasis - SPICE, IX Symposium Industrial Electronics INDEL 2012, Banja Luka / L. Gose. - November 1-3, pp. 6-21. (2012)

15. Sapozhnikov Val.V. Modeling ladder circuits / Val. V. Sapozhnikov, A. A. Lykov, A. V. Petrov, G. V. Osadchy // Ural Transport. -2007. - (3). - pp. 46-50. (2007),

16. Gorbachev A. M. Automation of analysis, examination and verification of technical documentation of the system of railway automation and telemechanics, A. M. Gorba $\neg$ chev // Izvestia Petersburg University of Railway Transport. -.2012. (4) pp. 73-78. (2012)

17. Smith D.J. Safety Critical Systems Handbook. A Straightforward Guide to Functional Safety, IEC 61508 and Related Standards, Including the Process IEC 61511 and Machinery IEC 62061 and ISO 13849 / D.J. Smith, Simpson Kenneth G.L. - Oxford, UK, Elsevier Ltd,. p 270.(2010)

18. Nazirjon, A., Dilshod, B., \& Davron, R. The problems of algorithmization of the automated system of accounting and control devices of railway automatics and telemechanics during high-speed movement. European science review, 1 (1-2). (2019).

19. Dilshod Baratov, Elmurod Astanaliev. Using innovative technologies of electronic technical document management for railway automation and telemechanics. International Engineering Journal for Research and Development. (5), pp. 5-9. (2020).

20. Dilshod Baratov, Elmurod Astanaliev. Methods of control of railway automation and telemechanics devices. International Scientific online Conference on Innovation in the modern education system, Washington University in St. Louis, USA. (1), pp. 77-80. (2020). 